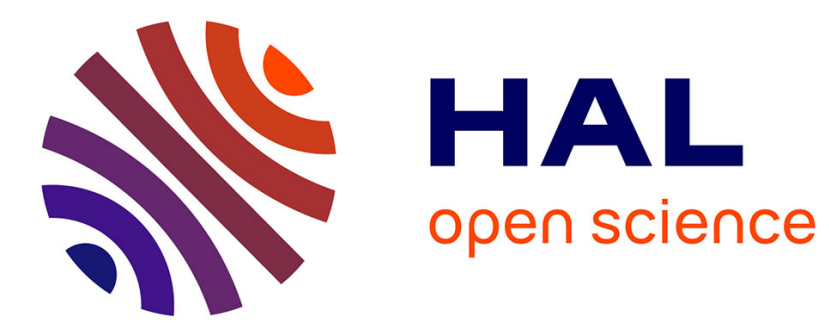

\title{
Individuality in Piano Performance Depends on Skill Learning
}

\author{
Baptiste Caramiaux, Frédéric Bevilacqua, Caroline Palmer, Marcelo \\ Wanderley
}

\section{- To cite this version:}

Baptiste Caramiaux, Frédéric Bevilacqua, Caroline Palmer, Marcelo Wanderley. Individuality in Piano Performance Depends on Skill Learning. 4th International Conference on Movement Computing MOCO 17, Jun 2017, London, United Kingdom. pp.1 - 7, 10.1145/3077981.3078046 . hal$01577872 \mathrm{v} 2$

\section{HAL Id: hal-01577872 \\ https://hal.science/hal-01577872v2}

Submitted on 22 Jul 2019

HAL is a multi-disciplinary open access archive for the deposit and dissemination of scientific research documents, whether they are published or not. The documents may come from teaching and research institutions in France or abroad, or from public or private research centers.
L'archive ouverte pluridisciplinaire $\mathbf{H A L}$, est destinée au dépôt et à la diffusion de documents scientifiques de niveau recherche, publiés ou non, émanant des établissements d'enseignement et de recherche français ou étrangers, des laboratoires publics ou privés. 


\section{Individuality in Piano Performance Depends on Skill Learning}

\author{
Baptiste Caramiaux \\ CIRMMT, McGill University, Montreal, Canada \\ UMR STMS Ircam-CNRS-UPMC, Paris, France \\ baptiste.caramiaux@ircam.fr \\ Caroline Palmer \\ Department of Psychology, McGill University \\ Montreal, Canada \\ caroline.palmer@mcgill.ca
}

\author{
Frédéric Bevilacqua \\ UMR STMS Ircam-CNRS-UPMC \\ Paris, France \\ frederic.bevilacqua@ircam.fr \\ Marcelo Wanderley \\ IDMIL - CIRMMT, McGill University \\ Montreal, Canada \\ marcelo.wanderley@mcgill.ca
}

\begin{abstract}
Expert musicians' performances embed a timing variability pattern that can be used to recognize individual performance. However, it is not clear if such a property of performance variability is a consequence of learning or an intrinsic characteristic of human performance. In addition, little evidence exists about the role of timing and motion in recognizing individual music performance. In this paper we investigate these questions in the context of piano playing. We conducted a study during which we asked non-musicians to perform a musical sequence at different speeds. Then we tested their learning performance at a fixed tempo. Focusing on the possibility to identify the participant based on performance features of timing and motion variability, we show that participant classification increases with practice. This suggests that 1) the individual timing signatures are affected by learning and 2) timing and motion variability is structured. Moreover, we show that motion features better classify individual performances than timing features.
\end{abstract}

\section{CCS CONCEPTS}

- Applied Computing $\rightarrow$ Psychology; Sound and music computing; • Computing methodologies $\rightarrow$ Machine learning approaches;

\section{KEYWORDS}

Motor Learning; Skill Acquisition; Music Performance; Classification; Individuality

\section{ACM Reference format:}

Baptiste Caramiaux, Frédéric Bevilacqua, Caroline Palmer, and Marcelo Wanderley. 2017. Individuality in Piano Performance Depends on Skill Learning. In Proceedings of 4th International Conference on Movement Computing, London, UK, June 2017 (MOCO'17), 7 pages.

https://doi.org/10.475/123_4

Permission to make digital or hard copies of part or all of this work for personal or classroom use is granted without fee provided that copies are not made or distributed for profit or commercial advantage and that copies bear this notice and the full citation on the first page. Copyrights for third-party components of this work must be honored. For all other uses, contact the owner/author(s).

MOCO'17, fune 2017, London, UK

(c) 2017 Copyright held by the owner/author(s).

ACM ISBN 123-4567-24-567/08/06 ..\$15.00

https://doi.org/10.475/123_4

\section{INTRODUCTION}

A fascinating feature of human perception of music is the ability to identify who is playing on the basis of acoustic features [5]. In other words, music carries identity information. Individuality in music performance has recently attracted the attention of researchers in psychology and musicology $[1,6,8]$. However, whether musical individuality is linked to mechanisms of skill acquisition remains largely unexplored.

The acquisition of motor skills is usually understood as the process by which a human is able to perform a motor task better, faster and more accurately $[3,14]$. In the process of acquiring new motor skills, practice plays a fundamental role [4], which differentiates this type of learning from changes in motor performance due to an external perturbation [10]. Learning performance is typically measured as a decrease of motor variability during practice [11]. But motor variability can also increase with skill acquisition through the development of a "behavioral repertoire" [13]. Music performance is an example involving the acquisition of complex motor skills, as one has to learn efficient movements and to perform these movements at the right time, and the development of an individual behavioral repertoire.

Perceptual recognition of individual performer stems from variability in acoustic features. On the one hand, performance variability can stem from musical expression, which "refers to the large and small variations in timing, intensity or dynamics, timbre, and pitch that form the microstructure of a performance and differentiate it from another performance of the same music" ([9], p118). On the other hand, performance variability can also stem from nondeliberate, subjective and consistent motion variations in space and time, which has been shown to be sufficient to accurately identify a performer above chance level [5]. Such variability is influenced by both biomechanical and cognitive factors in both space and time [7].

Stamatatos and Widmer [12] showed that machine learning can be used to identify the most likely performer given a set of music performances of the same piece represented through simple features of timing, articulations and dynamics, etc. Later Van Vugt et al. [15] examined to what extent only the note-by-note timing variability in piano performances can be used to recognize which performer played a given sequence. The authors termed this feature a "pianistic fingerprint". The authors showed that timing variability contains sufficient information to recognize expert pianists with high level of accuracy using a simple Bayesian classifier. Therefore, both of these 
studies suggest that timing variability in expert piano performance embeds an individual signature that can be used to recognize a piano performer.

Dalla Bella and Palmer [2] further showed that pianist fingers' velocity and acceleration information towards piano key were consistent enough (within participant) to be used to recognize which piano performer has played a given sequence of notes. Interestingly, they also showed that the classification accuracy is higher for the performers who had more musical training. In other words, this study suggests that motion kinematics involve an individual component that "crystalizes" with learning. However, all of their participants had at least several years of musical training, none of them were beginners, which makes difficult the analysis of the link between the existence of a participant-dependent signature in motion variability and learning. In a related study, Gingras et al. [5] also pointed out the link between performer identification and expertise. They showed that the best performers could be recognized with higher accuracy, suggesting a link between performer skill and musical individuality.

Here we want to examine the link between skill acquisition in piano and individual pianistic fingerprint in performance. Importantly, our approach does not rely on pianists with various levels of expertise but considers non-musicians, that is to say participants who never had any musical training.

The paper is structured as follows. In Section 2 we report on our data collection as part of a previous study. In the following section, we introduce the features used to characterize performance variability and the method used in the reported classification tests. In Section 3 we report the results and discuss them in the last section of the article.

\section{DATA COLLECTION}

The data reported here were collected as part of a study aiming at investigating the effect of a variable tempo learning schedule on timing skill acquisition with non-musician participants who learned an 8-note sequence on a piano keyboard. In this section, we do not provide the comprehensive details about the study, rather we report the essential elements of the method with regards to the machine classification of individual performers.

\subsection{Participants}

We recruited 48 non-musician participants (average of 22.5 years old, $\mathrm{SD}=3.4,25$ Female and 23 Male), all right-handed and none of them reporting any neurological condition. All participants signed a consent form approved by the University ethics committee.

\subsection{Materials and Equipment}

The participants' task was to learn the following 8-note sequence:

$$
C 3-G 3-E 3-B 3-D 4-F 4-B 4-F 5
$$

These 8 notes constituting the task were marked on the keyboard with colored tapes. Colors were used to indicate to the participant which finger to use to hit each key.

Participants were instructed to use a given fingering sequence to play the excerpt. The sequence of fingers was:

$$
F 1-F 3-F 2-F 4-F 1-F 2-F 3-F 4
$$

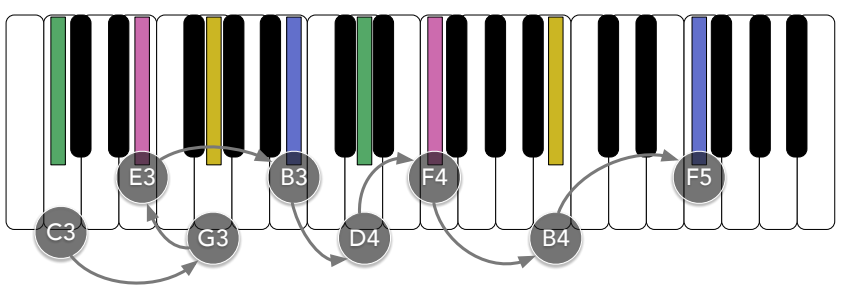

Figure 1: Motor task used in the study. The participants had to learn an 8-note sequence given by C3-G3-E3-B3-D4-F4-B4F5. The sequence had to be played using the following sequence of fingers: F1-F3-F2-F4-F1-F2-F3-F4 where F1: thumb, F2: index finger, F3: middle finger, and F4: ring finger.

where F1: thumb, F2: index finger, F3: middle finger, and F4: ring finger. In addition to the marking by colored tape, the fingering was explained by the experimenter at the beginning of the session. Figure 1 depicts the motor task.

Participants played on a Yamaha CP300 digital keyboard connected to a computer allowing for the recording of output MIDI data (pitches, onset times and velocities) and audio data. Participants' hand movements were recorded with a set of 8 infra-red cameras capturing markers placed on the hand. A total of 19 markers was used. One marker was placed on each finger's joint. One marker was placed on the top of the hand and two on the wrist. Markers on the keyboard have been placed in order to be able to rotate the data according to the same reference frame: the $\mathrm{x}$-axis is set along the keyboard (from grave to acute tones), the $y$-axis is in the transverse plane (forward direction from the participant perspective), and the $\mathrm{z}$-axis is the height.

In this paper we will consider only the markers placed at the fingertips of the first four fingers: thumb, index, middle and ring fingers.

\subsection{Procedure}

During the study, the participants had to play the sequence of notes a certain number of times (i.e. a certain number of trials). For each trial, the participant listened to an audio playback of the sequence of 8 notes played at a given tempo (audio stimulus synthesized using the same keyboard). Once the audio stimulus stopped, the participant had to replicate the sequence on the keyboard at the tempo of the stimulus. In addition, the participant was asked to perform the sequence as regular as possible in terms of tempo.

The study procedure had two main phases: a practice phase and a test phase.

2.3.1 Practice. Each participant went through a practice phase during which she performed 144 trials. Each trial had to be played at the tempo of the stimulus for that particular trial. The tempo of the stimulus (and so the stimulus itself) changed every 6 trials. The number of tempos practiced by a participant and the order of presentation of these tempos were the two factors tested in the study. More precisely we had two independent variables: TEMPO SET and the ORDER.

TEMPo SET is the set of tempos presented during the practice phase. Tempo Set can be: 
- Small: the tempo set is comprised of 2 tempos given by the following IOIs: $\{350 \mathrm{~ms}, 450 \mathrm{~ms}\}$

- Large: the tempo set is comprised of 6 tempos given by the following IOIs: $\{250 \mathrm{~ms}, 300 \mathrm{~ms}, 350 \mathrm{~ms}, 450 \mathrm{~ms}, 500 \mathrm{~ms}$, $550 \mathrm{~ms}\}$

ORDER is the order of presentation of the tempos within each tempo set. OrDER can be:

- Random: the order of the presentation is randomized

- Non-random: the order is repeated

Each participant was assigned randomly to one Tempo Set condition and one Order condition such that 12 participants were assigned to each combination of conditions (between-subjects design).

2.3.2 Test. After the practice phase, the participant started the test phase. During this phase, the participant had to perform 12 trials at three different tempos: $\mathrm{IOI}=400 \mathrm{~ms}, \mathrm{IOI}=350 \mathrm{~ms}$ and $\mathrm{IOI}=200 \mathrm{~ms}$.

This test phase was meant to assess the learning performance of each group at two untrained tempos (400ms and 200ms) and one trained tempo (350ms).

Our goal is to use these data to analyze to what extent we can recognize individual participants from their performance based on the motion and timing parameters of their performance.

\section{ANALYSIS}

We are interested in assessing the participants' performance variability and how it can be used to recognize each participant. From the data collected, we first characterize participants' variability during the performance through a set of features related to timing and motion. These features are then used as input data of a classifier to evaluate to what degree the participant can be recognized. Participant classification tests are performed at different learning stages (using data from the practice phase) and after learning (using data from the test phase).

\subsection{Features}

We consider two types of features characterizing participants' performance variability: timing variability features and motion features. The timing variability features account for temporal variations at each note onset across performances. The motion features account for temporal and spatial variability between two successive onsets across performances.

3.1.1 Timing variability features. Measures of timing variability stem from previous research in the field [15]. They are computed as follows: we extract the onset values of a participant's trial and we compute the difference between each onset value and the expected value of the onset as it would have been played perfectly at the prescribed tempo. As an example, during the test phase with $\mathrm{IOI}=400 \mathrm{~ms}$, the expected onset should occur every $400 \mathrm{~ms}$.

Figure 2 illustrates the timing variability feature. On the top panel, we report the inter-onset values of a trial by participant 3 during the test phase at $400 \mathrm{~ms}$, together with the expected (constant) inter-onset values. Deviations of the onsets from the expected onsets constitute a feature vector. On the bottom panel we reported the feature vectors from every trial performed by participant 3 during the test phase at $400 \mathrm{~ms}$.
Finally, each deviation is then divided by the expected inter-onset (e.g. $400 \mathrm{~ms}$ ) in order to have a measure independent of tempo.
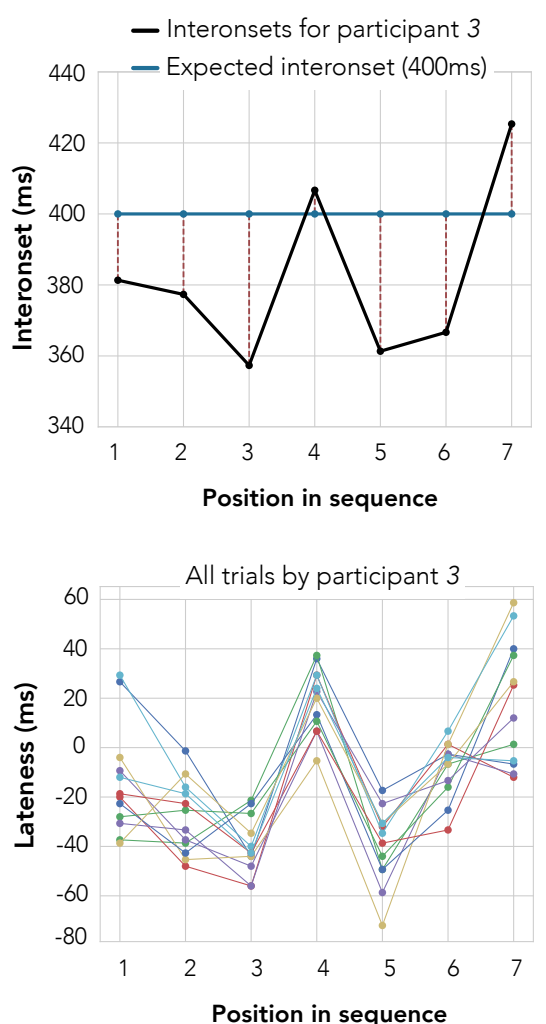

Figure 2: Timing variability features. The top panel shows the inter-onset values from one trial performed by one participant together with the expected inter-onset values. The bottom panel shows all the feature vectors for participant 3 during test phase at $400 \mathrm{~ms}$.

3.1.2 Motion variability features. Motion variability is derived from the motion kinematics of each marker, i.e. the marker positions, velocities and accelerations. Each marker is a 3-dimensional time series with sampling rate of $240 \mathrm{~Hz}$. We compute the velocity along each axis using a Savitzky-Golay filter with a 17-point window and an interpolation of order 3.

In Figure 3, we report an example. The top panel of the plot shows the evolution of the height of the index finger along time for participant 15 . The middle panel depicts the velocity computed from the finger's height and the bottom panel shows the acceleration profile.

Based on the motion kinematics, we consider only the fingers Attack. An attack is defined as the section between the finger press onset and the previous keypress. Keypresses are computed using the MIDI data: a keypress is detected at the velocity changes.

Previous work by Dalla Bella and Palmer have shown that the Attack embeds more information than the key release [2]. More precisely it has been shown that the velocity/acceleration profiles can be used to discriminate between participants. Figure 4 shows 


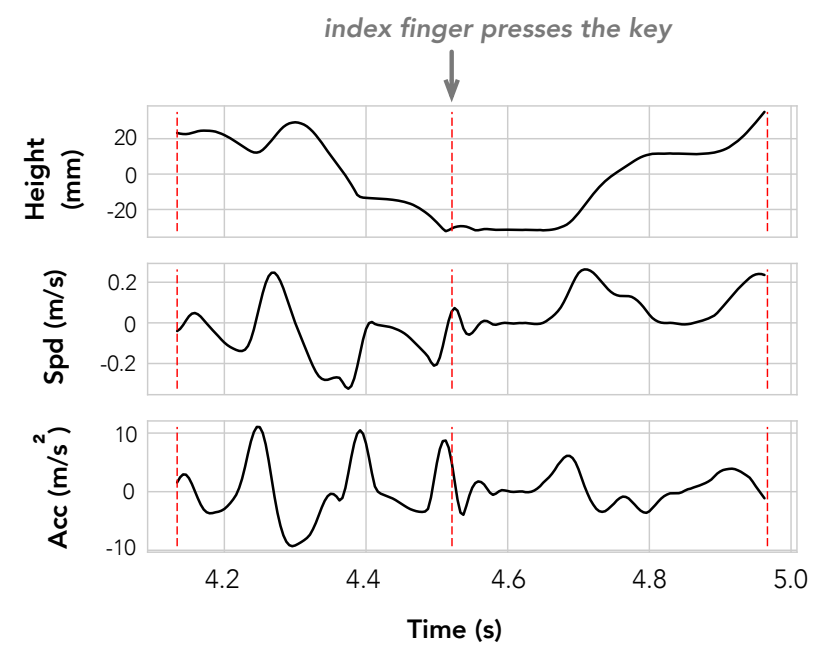

Figure 3: Motion kinematics. The top panel shows the evolution of the height of the index finger along time during for one participant. The middle panel depicts the velocity computed from the finger's height and the bottom panel shows the acceleration profile.

an example of the velocity/acceleration profile. This profile will be used as motion variability features for the classification.

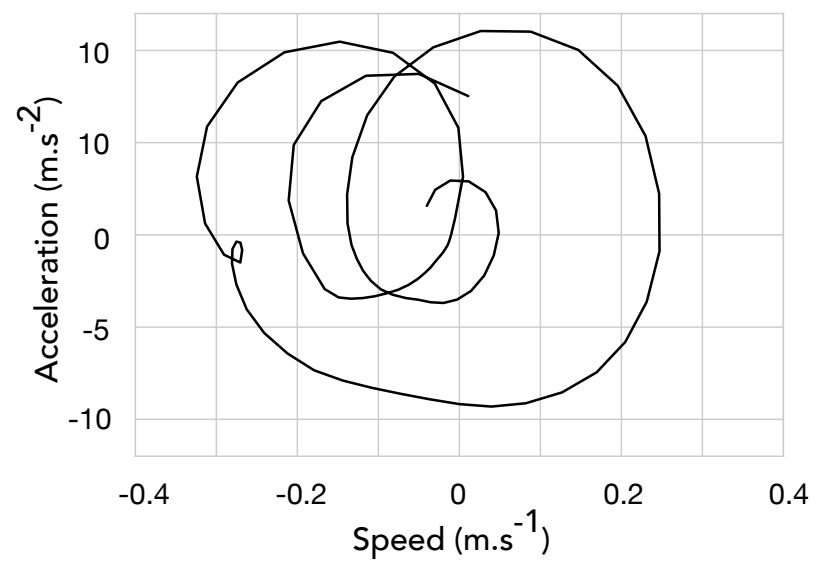

Figure 4: Velocity/acceleration profile of the index finger during Attack, ie before pressing the key.

\subsection{Classifiers}

Timing features are vectors whose components are considered as independent. We use a k-Nearest Neighbor to classify timing feature vectors as being produced by a given participant. This classifier has the advantage to involve one single parameter $\mathrm{k}$, the number of neighbors to be taken into account in the classification decision (in the following $\mathrm{k}=10$ ). Another motivation behind the choice of $\mathrm{k}-\mathrm{NN}$ is to be able to interpret the recognition outcomes: we want to be able to interpret the distance metric used by the classifier and its impact on the classification accuracy. In the implementation of the $\mathrm{kNN}$, the distance used is a Euclidean distance computed between feature vectors.

Regarding motion data however, we want to be able to interpret their variability in time and space and its impact on the classification. To do so we choose to use a classification based on Dynamic Time Warping (DTW). DTW has the advantage to return a distance value that takes into account the alignment cost between two timeseries that accounts for timing variability, and a geometric cost that accounts for spatial variability. The metric used to compute the distance between each motion velocity/acceleration values is Euclidean.

\subsection{Classification procedure}

3.3.1 Classification as a function of practice. Our first test is to evaluate the machine recognition accuracy of individual performers across practice and test phases. Specifically, we want to compare the classification accuracy at an early stage of practice to the classification accuracy at a late stage of practice and after practice (at test phase). This is made possible by creating a dataset of trials performed under the same task tempo. There are two tempos in common to every participant $(350 \mathrm{~ms}$, and $450 \mathrm{~ms})$ but only $350 \mathrm{~ms}$ is tested during the test phase.

We build three datasets: early stage, late stage, and after practice. The early-learning dataset is created by the 12 first trials at $350 \mathrm{~ms}$ for each participant (leading to $48 \times 12=576$ samples); the latelearning is formed by the 12 last trials at $350 \mathrm{~ms}$ (also leading to 48 $\mathrm{x} 12=576$ samples); the after-learning is created by the 12 trials at test tempo $350 \mathrm{~ms}$ ( 576 samples total). Figure 5 depicts the three datasets.

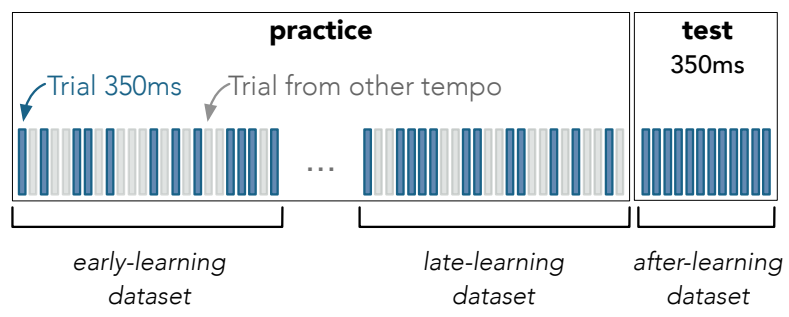

Figure 5: Creation of the datasets at early-stage of practice, late-stage of practice and after-practice (test phase). Each dataset is composed of 12 trials from each participant. Each trial has been performed in the tempo task of $350 \mathrm{~ms}$.

Classification tests are performed on these datasets using a leaveone-out cross-validation procedure. For k-NN, we train the classifier with 11 samples (11 trials) of each class (each participant) and we test with the remaining sample. We iterate this procedure 10 times. For DTW, we train the classifier with 1 sample (template-based) and with the test with another sample (randomly chosen). We iterate 10 times for each participant. 
3.3.2 Classification as a function of tempo after practice. The second test is to evaluate the machine recognition accuracy of individual performers across tempo conditions at the test phase. We remind the reader that, during the test phase, three tempos are tested: $400 \mathrm{~ms}, 350 \mathrm{~ms}$, and $200 \mathrm{~ms}$. For each test tempo we have 12 trials per participant. We create three datasets from these trials, one per test tempo. Therefore, each dataset has 576 samples. Classification tests are performed following the same leave-one-out procedure as described in the previous section.

\section{RESULTS}

In the following, the number of "classes" is 48 (the total number of participants in the study), which leads to a chance level of $2.1 \%$.

\subsection{Classification as a function of practice}

In this section, we aim to examine the role of practice on classification accuracy. To do so, we inspect the classification accuracies at an early stage of practice and at a late stage of practice and after practice (see previous section for details).

4.1.1 Timing. The first classification tests are performed using the timing variability features. Figure 6 reports the results.

First, we observe that the classification accuracies at all stages of practice are above chance level. In order to inspect the differences in participant classification accuracies according to the stage of practice, we perform a one-way analysis of variance with a factor Stage.

The ANOVA shows that the stage of practice has a significant effect on classification accuracies $(F(2,27)=29.2, p<0.001$, $\left.\eta^{2}=0.68\right)$. In addition, a Tukey's Honest Significant Test (HSD) test (with Bonferonni correction) shows that classification accuracy at the early stage of practice is significantly lower than classification accuracy at the late stage $(d \mu=+9.8 \%, p<0.001)$ and after practice $(d \mu=+11.1 \%, p<0.001)$. The post-hoc test also shows that classification accuracies at late stage and after practice are not significantly different.

4.1.2 Motion. We now consider motion features and we performed the second test of classification accuracy. Figure 7 shows that all classification accuracies are above chance level.

A one-way ANOVA shows that the stage of practice has a significant effect on participant classification accuracies based on motion features $\left(F(2,27)=25.7, p<0.001, \eta^{2}=0.66\right)$. A Tukey's HSD test (with Bonferonni correction) shows that classification accuracy at the early stage of practice is significantly lower than classification accuracy at late stage $(d \mu=+10.4 \%, p<0.001)$ and after practice $(d \mu=+16.6 \%, p<0.001)$. There is a marginal difference between classification accuracies at late stage and after practice $(d \mu=+6.1 \%$, $p=0.03)$.

4.1.3 Comparing participant classification accuracies based on timing and motion features. We assess the difference in classification accuracy between motion and timing features. We perform a two-way ANOVA with factors Stage and Feature (timing, motion features). The variable feature has a significant effect on classification accuracy $(F(1,54)=8.9, p<0.01)$, and there was no interaction between stage and feature. Classification accuracy is higher for motion features than timing features.

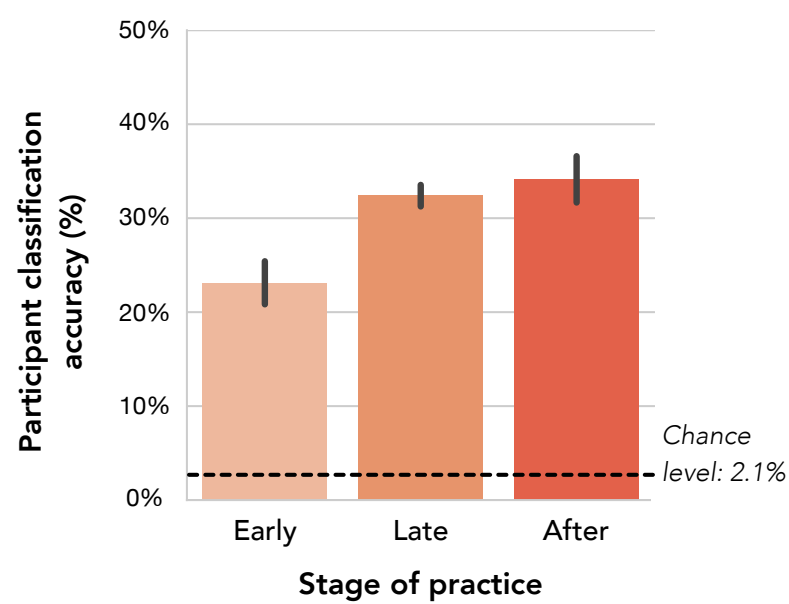

Figure 6: Mean classification accuracy (with 95\% confidence intervals $[\mathrm{CI}]$ ) using timing variability features at the beginning of practice, at the end of practice and at test (tempo 350ms).

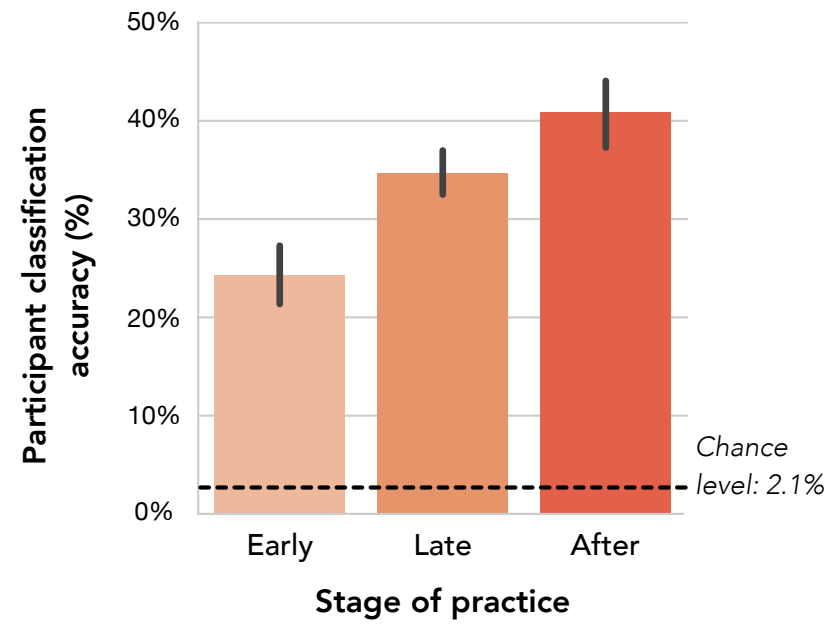

Figure 7: Mean classification accuracy (with 95\% CI) using the motion velocity/acceleration features at the beginning of practice, at the end of practice and at test (tempo $350 \mathrm{~ms}$ ).

\subsection{Classification as a function of tempo}

Now we evaluate the machine recognition accuracy of individual performers across tempo conditions at the test phase. Similarly, we performed classification tests on timing and motion features.

4.2.1 Timing. Classification accuracies at different test tempos, using timing features are depicted in Figure 8. The results show that the recognition accuracy is significantly above chance level.

A one-way ANOVA with factor Test Tempo shows that classification results differ significantly between test tempos $(F(2,27)=32.4$, 


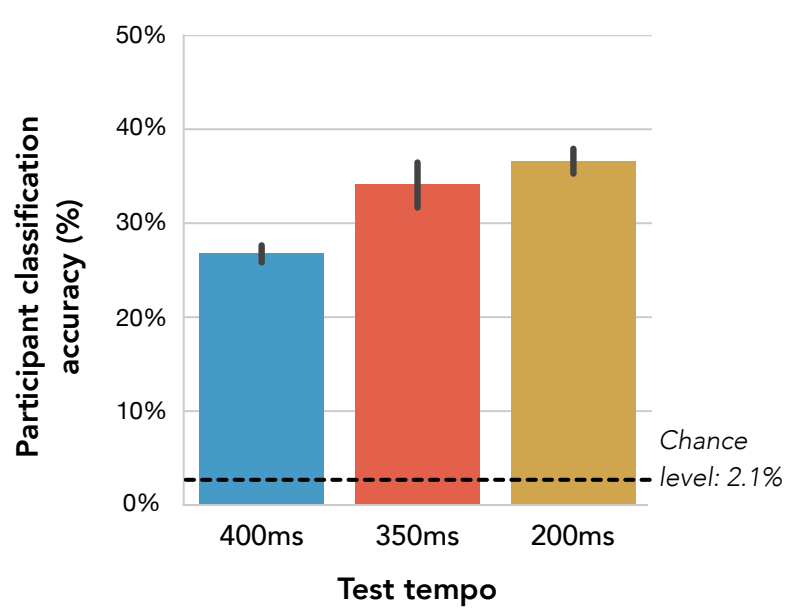

Figure 8: Mean classification accuracy (with 95\% CI) based on timing variability features at each test tempo: $400 \mathrm{~ms}, 350 \mathrm{~ms}$, 200ms.

$\left.p<0.001, \eta^{2}=0.7\right)$. A Tukey's HSD test, with Bonferonni correction, reveals that classification accuracy at test tempo $400 \mathrm{~ms}$ is lower than accuracy at test tempo $350 \mathrm{~ms}(d \mu=-7.4 \%, p<0.01)$ and at test tempo $200 \mathrm{~ms}(d \mu=-9.8 \%, p<0.001)$. Accuracies at test tempos $350 \mathrm{~ms}$ and $200 \mathrm{~ms}$ are not significantly different.

4.2.2 Motion. Finally, we test classification accuracies considering motion features. We examine the recognition accuracy in each test phase: Test Tempo 1 at $400 \mathrm{~ms}$, Test Tempo 2 at $350 \mathrm{~ms}$ and Test Tempo 3 at 200ms. Figure 9 reports the results.

As previously, classification accuracy is significantly higher than chance level. A one-way ANOVA with factor Test Phase reveals that there is no significant difference between classification scores obtained for each test tempo.

4.2.3 Comparing participant classification accuracies based on timing and motion features. Figures 8 and 9 suggest higher accuracy scores based on motion features than timing features. To examine if it is true, we perform a two-way ANOVA with factors Test Tempo and Feature (timing and motion). It reveals that the feature considered has a significant effect on classification accuracy $(F(1,54)=30.6, p<0.001)$, and there is an interaction between feature and test tempo $(p<0.001)$. A post-hoc analysis shows that classification accuracies differ between features for test tempos $400 \mathrm{~ms}$ and $350 \mathrm{~ms}$ but not $200 \mathrm{~ms}$. At test tempos $400 \mathrm{~ms}$ and $350 \mathrm{~ms}$, the participant classification mean accuracy is higher when considering motion features than timing variability features (resp. $d \mu=+12.1 \%, p<0.001$ and $d \mu=+6.7 \%, p<0.01)$.

\section{DISCUSSION}

We addressed whether musical individuality is linked to mechanisms of skill, a process by which a human is able to perform a task, better, faster and more accurately [11]. We know from previous works that piano experts can be identified based on their finger's velocity and acceleration profiles before hitting a key [2], or based

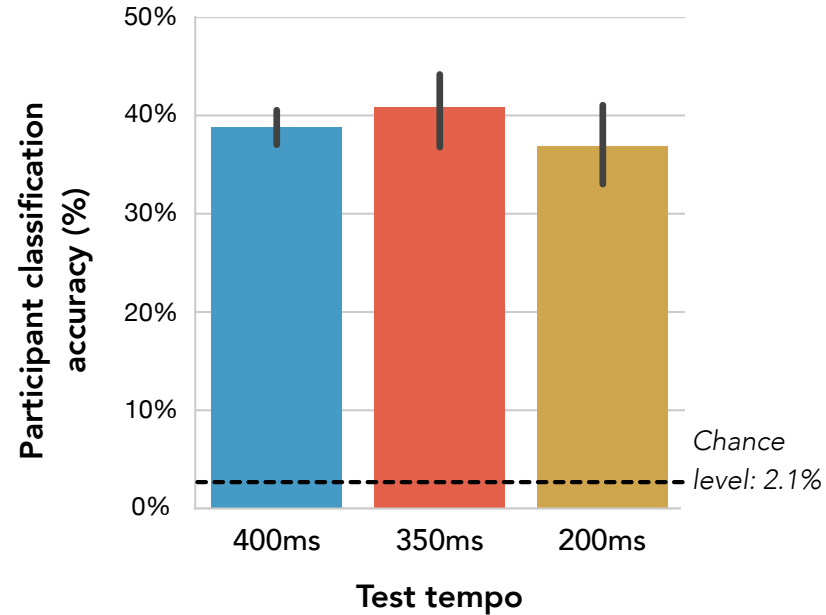

Figure 9: Mean classification accuracy (with 95\% CI) based on the motion velocity / acceleration features at each test tempo: $400 \mathrm{~ms}$, 350ms, $200 \mathrm{~ms}$.

on timing variability [15]. Our study with neophytes shows that machine recognition of individuals, computed from timing variability or motion features, improves significantly as the non-musicians practiced the task. This result suggests that the "personal pianistic fingerprint" $([2,15])$ is actually acquired through practice. In addition, classification accuracy at an early stage of practice is about ten times above chance level which means that participants possess an a priori "signature" in their movement kinematics. These results suggest that performance variability is structured and reflects individual characteristics that may "crystallize" in a stable form through practice.

The fact that we used template-based classification methods reinforces this interpretation. These methods involve non-linear decision surfaces whose complexity grows with the amount of data. The expected classification accuracy then improves, as the amount of data increases, if the between-class variability increases and the within-class variability decreases. In other words the expected classification accuracy improves as the class-specific templates stabilize around a certain pattern. In particular, we found in prior tests that parametric techniques, such as such as Support Vector Machines (SVM) or Neural Networks (as in [2]), return globally lower classification accuracy.

Participant classification accuracies reported in this paper are although lower than previous attempts in classifying individual performers based on motion, timing, or acoustic features [2, 5, 15]. Especially, Dalla Bella and Palmer [2] reached a very high classification accuracy using motion velocity and acceleration profiles towards keypress. We believe that three critical elements differentiate our study from this previous work. First, we shall stress the fact that our participants are neophytes (participants who never had any musical training) while previous studies considered participants with several years of musical training. Second, from a machine recognition perspective, our analysis involved data that have not been pre-processed. On the contrary, in the mentioned 
study [2], data used as input of the classifier are pre-processed with Functional ANOVA, to determine the region with the most between-subjects variance, and a Principal Component Analysis to reduce the number of dimensions of the input data. Finally, the number of participants to be classified (i.e. the number of classes) is higher (48) that in previous studies (4 pianists are considered in [2]).

Another finding is that participant classification accuracy based on timing variability is better during the test phase with fast tempo. At fast tempo, participants' movements are likely to be more ballistic than at slow tempo. In other words, movement control is mostly based on feed-forward control commands and relies less on feedback commands. The results presented in this paper then suggest that signature in timing variability may be linked to the feed-forward more than feedback processes [16, 17].

Our results also showed a difference in classification accuracies if considering motion features or timing features. Motion features globally led to higher accuracy than timing feature. It is not clear why motion features would lead to better results in the tests reported. One limitation of our study resides in the fact of considering only one sequence in order to compare timing and motion variability as input of a machine-based participant recognition method. Further comparisons of motion and timing features in terms of classification accuracy might be made by considering a variety of sequences which would require a combination of finger movements more complex that those used here [2, 7].

Finally, and importantly, in this work we did not aim at proposing a method to identify players with the highest accuracy. Rather, we highlight the possibility to use machine recognition method as a means to study parameters of skill acquisition in music performance. This research direction has yet to be mined.

\section{ACKNOWLEDGMENTS}

This project has received funding from the European Union?s Horizon 2020 research and innovation programme under the Marie Sklodowska-Curie grant agreement No 659232.

\section{REFERENCES}

[1] Michel Bernays and Caroline Traube. 2014. Investigating pianists' individuality in the performance of five timbral nuances through patterns of articulation, touch, dynamics, and pedaling. Individuality in music performance (2014), 35.

[2] Simone Dalla Bella and Caroline Palmer. 2011. Rate effects on timing, key velocity, and finger kinematics in piano performance. PloS one 6, 6 (2011), e20518.

[3] Jörn Diedrichsen and Katja Kornysheva. 2015. Motor skill learning between selection and execution. Trends in cognitive sciences 19, 4 (2015), 227-233.

[4] K Anders Ericsson, Ralf T Krampe, and Clemens Tesch-Römer. 1993. The role of deliberate practice in the acquisition of expert performance. Psychological review 100, 3 (1993), 363.

[5] Bruno Gingras, Tamara Lagrandeur-Ponce, Bruno L Giordano, and Stephen McAdams. 2011. Perceiving musical individuality: performer identification is dependent on performer expertise and expressiveness, but not on listener expertise. Perception 40, 10 (2011), 1206-1220.

[6] Réka Koren and Bruno Gingras. 2014. Perceiving individuality in harpsichord performance. Individuality in music performance (2014), 84.

[7] Janeen D Loehr and Caroline Palmer. 2007. Cognitive and biomechanical influences in pianists' finger tapping. Experimental brain research 178, 4 (2007), 518-528.

[8] Manuela M Marin and Joydeep Bhattacharya. 2013. Getting into the musical zone: trait emotional intelligence and amount of practice predict flow in pianists. (2013).

[9] Caroline Palmer. 1997. Music performance. Annual review of psychology 48, 1 (1997), 115-138.
[10] Reza Shadmehr, Maurice A Smith, and John W Krakauer. 2010. Error correction, sensory prediction, and adaptation in motor control. Annual review of neuroscience 33 (2010), 89-108.

[11] Lior Shmuelof, John W Krakauer, and Pietro Mazzoni. 2012. How is a motor skill learned? Change and invariance at the levels of task success and trajectory control. Journal of neurophysiology 108, 2 (2012), 578-594.

[12] Efstathios Stamatatos and Gerhard Widmer. 2005. Automatic identification of music performers with learning ensembles. Artificial Intelligence 165, 1 (2005), 37-56.

[13] Nicholas Stergiou and Leslie M Decker. 2011. Human movement variability, nonlinear dynamics, and pathology: is there a connection? Human movement science 30, 5 (2011), 869-888.

[14] Sebastian Telgen, Darius Parvin, and Jörn Diedrichsen. 2014. Mirror reversal and visual rotation are learned and consolidated via separate mechanisms: recalibrating or learning de novo? The fournal of Neuroscience 34, 41 (2014), 13768-13779.

[15] Floris Tijmen van Vugt, Hans-Christian Jabusch, and Eckart Altenmüller. 2013. Individuality that is unheard of: systematic temporal deviations in scale playing leave an inaudible pianistic fingerprint. (2013).

[16] Daniel M Wolpert, Jörn Diedrichsen, and J Randall Flanagan. 2011. Principles of sensorimotor learning. Nature Reviews Neuroscience 12, 12 (2011), 739-751.

[17] Daniel M. Wolpert, Zoubin Ghahramani, and Michael I. Jordan. 1995. An internal model for sensorimotor integration. Science 269 (1995), 1880-1882. 